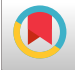

\title{
Prevalence of Anti-Toxoplasma gondii Antibodies in Young Iranians: The CASPIAN III Study
}

\author{
Mohammad Javad Gharavi, ${ }^{1,2}$ Mona Roozbehani, ${ }^{2}$ Abolfazl Miahipour, ${ }^{3}$ Mojgan Oshaghi, ${ }^{4}$ Behnaz \\ Gharegozlou, ${ }^{5}$ Enayatollh Kalantar, ${ }^{6}$ Shervin Ghaffari Hoseini, ${ }^{7}$ Nasser Mostafavi, ${ }^{7}$ Ramin Heshmat, ${ }^{8}$ \\ Abdolhossien Naseri, ${ }^{5}$ Mostafa Qorbani, ${ }^{9,10,}$ and Roya Kelishadi ${ }^{11}$ \\ ${ }^{1}$ Department of Medical Parasitology, Deputy of Researches and Technologies, Alborz University of Medical Sciences, Karaj, IR Iran \\ ${ }^{2}$ Department of Parasitology, School of Medicine, Iran university of medical sciences,Tehran, IR Iran \\ ${ }^{3}$ Department of Medical Parasitology and Mycology, School of Medicine, Alborz University of Medical Sciences, Karaj, IR Iran \\ ${ }^{4}$ Department of Microbiology, Faculty of Allied Medicine, Iran University of Medical Sciences, Tehran, IR Iran \\ ${ }^{5}$ Department of Immunology, Faculty of Allied Medicine, Iran University of Medical Sciences, Tehran, IR Iran \\ ${ }^{6}$ Department of Microbiology and Immunology, School of Medicine, Alborz University of Medical Sciences, Karaj, IR Iran \\ ${ }^{7}$ Infectious Diseases and Tropical Medicine Research Center, Isfahan University of Medical Sciences, Isfahan, IR Iran \\ ${ }^{8}$ Chronic Disease Research Center, Endocrinology and Metabolism Population Sciences Institute, Tehran University of Medical Sciences, Tehran, IR Iran \\ ${ }^{9}$ Non-communicable Diseases Research Center, Alborz University of Medical Sciences, Karaj, IR Iran \\ ${ }^{10}$ Endocrinology and Metabolism Research Center, Endocrinology and Metabolism Clinical Sciences Institute, Tehran University of Medical Sciences, Tehran, IR Iran \\ ${ }^{11}$ Child Growth and Development Research Center, Research Institute for Primordial Prevention of Non-communicable Disease, Isfahan University of Medical Sciences, \\ Isfahan, IR Iran \\ "Corresponding author: Dr. Mostafa Qorbani, Non-communicable Diseases Research Center, Alborz University of Medical Sciences, Karaj, IR Iran. E-mail: \\ mqorbani1379@yahoo.com
}

Received 2016 October 01; Revised 2017 March 25; Accepted 2017 April 01.

\begin{abstract}
Background: Toxoplasmosis is a worldwide neglected tropical and sub-tropical infection caused by Toxoplasma gondii (T. gondii). Most of the previous studies on the seroprevalence of T. gondii in Iran have been done at provincial level and on specific populations, including pregnant females. Socioeconomic parameters are associated with the prevalence of this disease. In this study, the authors evaluated the presence of anti-T. gondii antibodies and the related risk factors in Iranian adolescents.

Methods: This was a cross-sectional study on serum samples from the third Iranian national school-based survey (the CASPIAN III study), which included 10- to 18-year-old students. Participants were selected by multistage sampling from 27 Iranian provinces. In this study, serum samples of 882 adolescents from 16 provinces were randomly selected and examined for IgG and IgM antibodies against T. gondii by the Enzyme Linked Fluorescent Assay(ELFA). Demographic and socioeconomic factors related to T. gondii infection were gathered using the global school-based health survey (GSHS) questionnaire.

Results: The overall T. gondii IgG and IgM seropositivity was 56.3\% (95\% CI:53.4 to 59.2) and 3.7\% (95\%CI: 2.7 to 4.9), respectively. In multivariate logistic regression model, family size was statistically associated with seroprevalence of anti T. gondii IgG. Living in crowded households (households with more than 4 people vs. households with less than 4 people) increased the risk of seropositivity of $T$. gondii (OR:1.40, 95\% CI:1.10 to 1.99).

Conclusions: The results of this study indicate that about $40 \%$ of Iranian adolescents have not had contact with the T. gondii, thus the risk of congenital toxoplasmosis might be high in young females. Also, household size was the main factor associated with $T$. gondii infection. Preventive strategies and health education in Iranian adolescents are recommended.
\end{abstract}

Keywords: Toxoplasmosis, Seroprevalence, Risk Factor, Iran

\section{Background}

Toxoplasmosis is a worldwide neglected tropical and sub-tropical infection caused by the intracellular protozoan, called Toxoplasma gondii (1). Infection with T. gondii does not cause important disease in immunologically normal hosts, however, it can be fatal in immunocompromised individuals. Congenital toxoplasmosis is also an important health issue worldwide(2). The parasite completes its life cycle in cat's intestine and excreted infective oocytes contaminate water and vegetables, and their ingestion can infect humans. Eating tissue cysts in raw meat is another way of human infection $(3,4)$.

Epidemiological evidence shows that one-third of the world population has been in contact with T. gondii, however, the infection rate varies greatly by country, ranging from $20 \%$ to $80 \%$ (5-7). Prevalence of $T$. gondii in different regions is mainly dependent on culture, eating habits, and 
climate. For example, ingestion of undercooked meat is routine in France, leading to a high seroprevalence in this country $(8,9)$. In Latin America or sub-Saharan Africa, tropical climate facilitates survival of oocytes in the environment, thus the prevalence is high $(10,11)$. Toxoplasmosis is prevalent in Iran and various seroprevalence rates have been reported from different regions of the country, which might be due to the environmental, socio-economical, and cultural differences in this country (12).

Although previous studies in Iran have reported seroprevalence of $T$. gondii in some specific groups, including pregnant females and youth, most of them have been done at provincial level (13-19) and studies at national level are scare. This study was designed to evaluate the frequency of anti-T. gondii antibodies and related risk factors in a representative sample of Iranian adolescents.

\section{Methods}

\subsection{Patients and Setting}

The present study was designed as a cross sectional study, which was run on representative data of the national surveillance study, the CASPIAN III (2009 to 2010). The aims and details of methods have been reported previously (20).

In this nationwide survey, 5528 students (10- to 18-yearold) were selected by multistage random sampling. Overall, 27 out of 30 Iranian provinces took part in this study. Sample size in each province was proportionate to the population of that province. In the first stage, schools were randomly selected from lists provided by the information bank of the Ministry of Education and thereafter students were randomly enrolled. Stratified sampling was applied based on the level of school (elementary, secondary, and high school) and the living area (urban vs. rural) with equal gender ratios. One of the parents completed a questionnaire containing demographic and socioeconomic factors under supervision of one of the team members and also signed a written informed consent for blood sampling and use of data for research. Blood samples were obtained from students and serum samples were stored at $-70^{\circ} \mathrm{C}$ for further use.

The researchers aimed to determine the seroprevalence of $T$. gondii in 3 age groups (10 to 12, 13 to 15, and 16 to 18 ) and both genders of Iranian adolescents; as the estimated prevalence was $40 \%$ (21), the researchers needed a sample size of about 900 to obtain a $95 \%$ confidence interval and $10 \%$ precision in the reported prevalence. A total of 900 residual serum samples of CASPIAN III from 16 provinces were selected randomly and were used in this study along with their questionnaires. Only 882 samples were available, because of depletion of samples in previous studies.

\subsection{Antibody Detection}

IgG and IgM antibodies against T. gondii were screened by the Enzyme Linked Fluorescent Assay (ELFA) method. The test was performed on a fully automated Vitek immuno diagnostic assay system (VIDAS), as described by the manufacturer. The anti-Toxo IgG and IgM (bioMérieux, Marcy l'Etoile, France) immunoassays kit was used in this study. IgG titers of $\geq 4 \mathrm{IU} / \mathrm{mL}$ and IgM titers $\geq 0.65 \mathrm{IU} / \mathrm{mL}$ were considered as positive test results (22).

The researchers used data from CASPIAN III study to record age, gender, residency (urban/rural), and socioeconomic parameters of the participants. Number of households, parents' education, and parents' occupation were included in the analysis, independently; however, variables such as having a private car, school type (public/private), type of home (private/rented) and having personal computer were summarized in a variable named family socioeconomic status (SES). The method and variables used for calculating family SES were initially approved in the Progress International Reading Literacy Study (PIRLS) for Iran (23). The parameters were given scores and total scores were regarded as the SES score, which was rated as low, moderate, and high. Related national ethical committees approved the CASPIAN III study and Alborz University of Medical Sciences ethical committee accepted this survey.

\subsection{Statistical Analysis}

Seroprevalence of $T$. gondii is presented with $95 \%$ confidence interval (CI). Seroprevalence of $T$. gondii across demographic characteristics was compared using the Chisquare test. Association of T. gondii seropositivity with demographic characteristics was assessed using univariate and multivariate logistic regression analysis. Results of logistic regression analysis was reported as odds ratio (OR) and 95\% confidence interval (CI). Significance level of Pvalue was set at 0.05 .

\section{Results}

Of the 900 students aged 10 to 18 years, who were selected for the CASPIAN III study, samples from 882 (48.3\% female) students were checked for anti-T. gondii antibodies. The distribution of participants in different age groups, residence areas, genders, and SES levels were nearly equal to the CASPIAN III data.

Mean age of participants (14.82 \pm 2.77 years) was not different between males $(14.78 \pm 2.88)$ and females $(14.86 \pm$ 2.65). Overall, $61.52 \%$ of participants were from metropolitan areas and $38.42 \%$ were from non-metropolitan regions. 
Distribution of students in school levels was $31.75 \%$ in primary, $41.72 \%$ in secondary, and $26.53 \%$ in high school. The overall T. gondii IgG seropositivity was 56.3\% (95\%CI: 53.4 to 59.2). The T. gondii IgG serpositivity in males (57.96\%) and females (54.78\%) was not statistically significant. Table 1 displays the seropositivity of $T$. gondii IgG, according to students' demographic characteristics.

Table 1. Seroprevalence of Toxoplasma gondii in Iranian Adolescents According to Demographic Characteristics: the CASPIAN III Study ${ }^{a}$

\begin{tabular}{|c|c|c|}
\hline & $\%$ & $95 \% \mathrm{CI}$ \\
\hline Entire study population & 56.34 & $53.39-59.24$ \\
\hline \multicolumn{3}{|l|}{ Age group, $y$} \\
\hline $10-12$ & 58.56 & $53.83-63.15$ \\
\hline $13-15$ & 55.86 & $50.65-60.94$ \\
\hline $15-18$ & 55.51 & $50.79-60.14$ \\
\hline Pvalue & \multicolumn{2}{|c|}{0.65} \\
\hline \multicolumn{3}{|l|}{ Gender } \\
\hline Male & 57.96 & $52.62-63.12$ \\
\hline Female & 54.78 & $50.03-59.46$ \\
\hline Pvalue & \multicolumn{2}{|c|}{0.42} \\
\hline \multicolumn{3}{|l|}{ Residence area } \\
\hline Metropolitan & 55.72 & $52.66-58.73$ \\
\hline Non-metropolitan & 57.1 & $51.52-62.5$ \\
\hline Pvalue & \multicolumn{2}{|c|}{0.64} \\
\hline \multicolumn{3}{|l|}{ Number of households } \\
\hline 4 & 52.96 & $47.9-57.96$ \\
\hline$>4$ & 58.51 & $54.95-61.99$ \\
\hline Pvalue & \multicolumn{2}{|c|}{0.1} \\
\hline \multicolumn{3}{|l|}{ SES } \\
\hline Low & 53.02 & $46.61-59.34$ \\
\hline Mod & 61.42 & $55.62-66.92$ \\
\hline High & 54.78 & $50.04-59.43$ \\
\hline Pvalue & \multicolumn{2}{|c|}{0.11} \\
\hline \multicolumn{3}{|l|}{ Father education } \\
\hline Illiterate & 58.28 & $51.24-65$ \\
\hline Under diploma & 56.72 & $51.22-62.07$ \\
\hline Diploma and academic & 55.56 & $50.32-60.67$ \\
\hline Pvalue & \multicolumn{2}{|c|}{0.84} \\
\hline \multicolumn{3}{|l|}{ Mother education } \\
\hline Illiterate & 54.47 & $48.59-60.23$ \\
\hline Under diploma & 59.38 & $54.68-63.92$ \\
\hline Diploma and academic & 50.37 & $41.7-59.01$ \\
\hline Pvalue & \multicolumn{2}{|c|}{0.15} \\
\hline \multicolumn{3}{|l|}{ Mother occupation } \\
\hline Employed & 43.86 & $30.29-58.41$ \\
\hline Housewife & 57.16 & $54.58-59.71$ \\
\hline Pvalue & \multicolumn{2}{|c|}{0.07} \\
\hline \multicolumn{3}{|l|}{ Father occupation } \\
\hline Unemployed & 58.82 & $46.17-70.41$ \\
\hline Employed/office work & 54.18 & $49.69-58.61$ \\
\hline Self employed & 58.43 & $52.16-64.43$ \\
\hline Pvalue & 0.48 & \\
\hline
\end{tabular}

The overall T. gondii IgM seropositivity was 3.7\% (95\%CI: 2.7 to 4.9 ). The T. gondii IgM seropositivity in males (3.48\%) and females (3.83\%) was not statistically different (P value: 0.81 ). Figures 1 and 2 show the seroprevalence of $T$. gondii IgG and IgM, according to age groups. The seroprevalence of T. gondii IgG and IgM was not statistically different across age groups.



Figure 1. Seroprevalence of Toxoplasma gondii IgG According to Age in Iranian Adolescents: the CASPIAN III Study

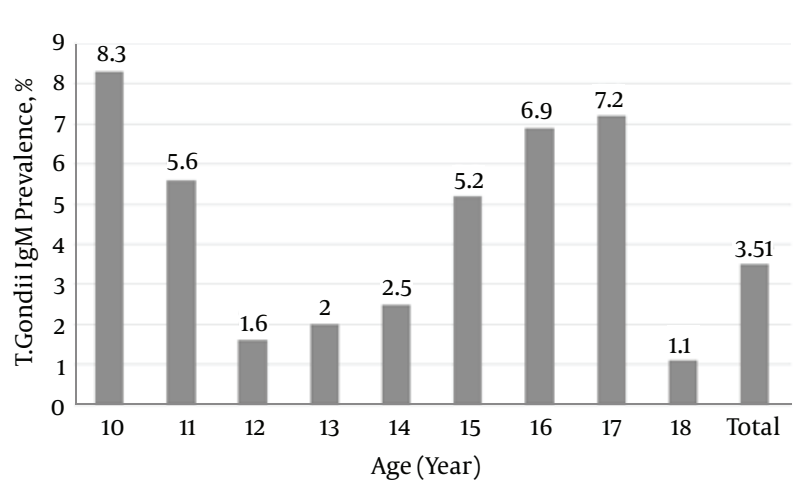

Figure 2. Seroprevalence of Toxoplasma gondii IgM by Age in Iranian Adolescents: the CASPIAN III Study

Table 2 shows the association of demographic characteristics and T. gondii IgG seroprevalence in univariate and multivariate logistic regression analysis. In univariate model, the SES of family was associated with seroprevalence of $T$. gondii. In multivariate model, number of household members was statistically associated with seroprevalence of $T$. gondii. Living in a crowded household (households with more than 4 people compared to households with less than 4 people) increased the risk of seropositivity of T. gondii (OR:1.40, 95\% CI:1.10 to 1.99). 
Table 2. The Association of Toxoplasma gondii Seropositivity with Independent Variables in Logistic Regression Analysis: the CASPIAN III Study

\begin{tabular}{|c|c|c|c|c|c|}
\hline & & \multicolumn{2}{|c|}{ Crude } & \multicolumn{2}{|c|}{ Adjusted } \\
\hline & & OR $(95 \% \mathrm{CI})$ & P Value & $\mathrm{OR}(95 \% \mathrm{CI})$ & PValue \\
\hline \multirow{3}{*}{ Age group, $y$} & $10-12$ & Reference & . & - & . \\
\hline & $13-15$ & $0.88(0.62-1.24)$ & 0.47 & $0.86(0.59-1.24)$ & 0.42 \\
\hline & $15-18$ & $0.86(0.59-1.24)$ & 0.41 & $0.77(0.51-1.16)$ & 0.22 \\
\hline \multirow{2}{*}{ Number of households } & $<4$ & Reference & - & - & . \\
\hline & $>4$ & $1.26(0.95-1.70)$ & 0.09 & $1.40(1.1-1.99)$ & 0.04 \\
\hline \multirow{2}{*}{ Gender } & Male & Reference & - & - & . \\
\hline & Female & $0.87(0.63-1.22)$ & 0.42 & $0.86(0.64-1.14)$ & 0.3 \\
\hline \multirow{3}{*}{ SES } & Low & Reference & - & - & - \\
\hline & Moderate & $1.41(1.01-1.95)$ & 0.03 & $1.34(0.95-1.93)$ & 0.07 \\
\hline & High & $1.07(0.75-1.53)$ & 0.68 & $1.22(0.85-1.22)$ & 0.23 \\
\hline \multirow{2}{*}{ Residence area } & Metropolitan & Reference & - & - & - \\
\hline & Non-metropolitan & $1.05(0.82-1.36)$ & 0.64 & $1.01(0.74-1.37)$ & 0.94 \\
\hline
\end{tabular}

Abbreviation: SES, socioeconomic status.

\section{Discussion}

This study revealed a seroprevalence of $56.3 \%$ and $3.7 \%$ for IgG and IgM antibodies against T. gondii in Iranian adolescent, respectively. As shown by Figure 1, seroprevalence of $T$. gondii was not much different for participants of different ages (ranging from about 50\% to 60\%). This might propose that the infection is acquired early and almost before the age of 10 in Iranians.

Studies have been performed to evaluate T. gondii infection in pregnant females or females of the childbearing age in some countries and different seroprevalence rates were estimated. The reported seroprevalence of $T$. gondii infection was $71 \%$ in France $(8,9)$. Prevalence of $T$. gondii antibodies was 78\% in Nigerian pregnant females (11), 22.5\% in the population of United States (24), 92.5\% in Ghana (25), 43\% in Austria (26), 22.1\% in Slovakia (27), 30\% in Spain (28), 24.6\% in Turkey (29), and 59\% in Argentina (30).

The current found an overall T. gondii seroprevalence of $60 \%$ in the studied adolescents, which indicates that $T$. gondii prevalence in Iran is not as much as the prevalence in countries, such as France, Ghana, and Nigeria (8, 9, 25, 31-33).

In Iran, the prevalence rates of anti-toxoplasma were highly variable. The current findings are consistent with prior reports on toxoplasmosis in several provinces of Iran. Previous studies reported that $71 \%$ and $55.7 \%$ of subjects in Sari and the Caspian Sea area were infected with T. gondii, respectively $(18,34)$.

This study investigated the household and individuallevel risk factors for toxoplasmosis. Results of the present study show that only household size was associated with toxoplasmosis. In fact, demographic factors such as age, gender, residence area, parents' education, father's job, and socioeconomic status were not associated with toxoplasmosis. The researchers found that children living in crowded households (households with more than 4 people) were more likely to have positive $T$. gondii test results than those with a small household size (households with less than 4 people); as the parasite is not transmitted by direct human contact, the reason for this association is not clear and it might be related to other risk factors, such as food hygiene and nutritional habits, which were not assessed in this study.

Several studies in Iran and other countries have assessed the risk factors for toxoplasmosis; previous systematic review and meta-analysis studies in Iran, which were conducted on risk factors of toxoplasmosis in pregnant females and general population show that the place of residence and keeping pets were 2 significant risk factors of toxoplasmosis $(21,35)$. In the general population, having direct contact with cats, consumption of uncooked meat and raw fruits or vegetables, low level of education, and living in rural areas were the main risk factors of toxoplasmosis (21). This study, in line with the study of Gyang et al. could not find any associations between age, gender, place of residence, and parents' occupation/educational level with toxoplasmosis (36). In another study in Turkey, the researchers did not find any relationship between $T$. gondii prevalence and family size, level of education, and socioeconomics of the family (37). The reason for these controversies is not obvious; different target populations with diverse cultures and different study designs with regard to the included risk factors might stand for these discrepancies.

Strengths and limitations: The data of the present study was obtained from a representative nationwide sur- 
vey on adolescents, which increases generalizability of the findings. It was not possible to include all Iranian provinces in this study, which may decrease generalizability of findings. In addition, the researchers were not able to assess probable risk factors of $T$. gondii infection, including keeping a pet, consuming raw or undercooked meat and unwashed vegetables and fruits.

Conclusion: The results of this study indicate that about $40 \%$ of Iranian adolescent have not had contact with the $T$. gondii, thus a considerable proportion of young females are at risk of congenital toxoplasmosis. Also, household size (living in a crowded family) was the main risk factor of T. gondii infection. Preventive strategies and health education for Iranian adolescents are recommended.

\section{Acknowledgments}

The authors gratefully acknowledge the team of the CASPIAN-III study for providing the serum samples and demographic data for this study.

\section{Footnote}

\section{Conflict of Interest: None.}

\section{References}

1. Furtado JM, Smith JR, Belfort RJ, Gattey D, Winthrop KL. Toxoplasmosis: a global threat. J Glob Infect Dis. 2011;3(3):281-4. doi: 10.4103/0974777X.83536. [PubMed: 21887062].

2. Boyle JP, Radke JR. A history of studies that examine the interactions of Toxoplasma with its host cell: Emphasis on in vitro models. Int J Parasitol. 2009;39(8):903-14. [PubMed: 19630139].

3. Montoya JG, Liesenfeld O. Toxoplasmosis. Lancet. 2004;363(9425):1965-76. doi: 10.1016/S0140-6736(04)16412-X. [PubMed: 15194258].

4. Dubey JP. Toxoplasmosis - a waterborne zoonosis. Vet Parasitol. 2004;126(1-2):57-72. doi: 10.1016/j.vetpar.2004.09.005. [PubMed: 15567579].

5. Pappas G, Roussos N, Falagas ME. Toxoplasmosis snapshots: global status of Toxoplasma gondii seroprevalence and implications for pregnancy and congenital toxoplasmosis. Int $J$ Parasitol. 2009;39(12):1385-94. doi: 10.1016/j.ijpara.2009.04.003. [PubMed: 19433092].

6. Tenter AM, Heckeroth AR, Weiss LM. Toxoplasma gondii: from animals to humans. Int J Parasitol. 2000;30(12-13):1217-58. [PubMed: 11113252].

7. Shahmoradi A, Rezaeian M, Dalimi ASLE A. Sheep-an important reservoir of human toxoplasmosis in iran. Med J Islamic Republic Iran. 1993;7(3):173-4.

8. Jeannel D, Niel G, Costagliola D, Danis M, Traore BM, Gentilini M. Epidemiology of toxoplasmosis among pregnant women in the Paris area. Int J Epidemiol. 1988;17(3):595-602. [PubMed: 3264821].

9. Ancelle T, Goulet V, Tirard-Fleury V, Baril L, Du Mazaubrun C, Thulliez PH, et al. La Toxoplasmose chez la femme enceinte en France en 1995. Resultats d'une enquete nationale perinatale. Bull Epidemiol Hebd.1996;51:227-9.

10. Schwartzman JD, Maguire JH. Tropical infectious diseases: principles, pathogens, and practice. In: Guerrant RC, Walker DH, Weller PF, editors. Philadelphia: Churchill Livingstone; 1999. Systemic coccidia (toxoplasmosis); p. 829-39.
11. Onadeko MO, Joynson DH, Payne RA. The prevalence of Toxoplasma infection among pregnant women in Ibadan, Nigeria. J Trop Med Hyg. 1992;95(2):143-5. [PubMed: 1560485].

12. Martin PA, Bouza PE. Infect Dis. In: Choen J, Powderly WG, editors. 2004. Blood and Tissue Protozoa; p. 2448-50.

13. Rostami A, Keshavarz H, Shojaee S, Mohebali M, Meamar AR. Frequency of Toxoplasma gondii in HIV Positive Patients from West of Iran by ELISA and PCR. Iran J Parasitol. 2014;9(4):474-81. [PubMed: 25759728].

14. Keshavarz H, Horri H. Prevalence of Toxoplasma gondii in pregnant women in kerman. J Med Council.1995;13:322-8.

15. Heidari A. Seroepidemiological study of toxoplasmosis among highschool girls in Gonabad. Qom Univ Med Sci J. 2012;5(3).

16. Abdi J, Shojaee S, Mirzaee A, Keshavarz H. Seroprevalence of toxoplasmosis in pregnant women in Ilam province, Iran. Iran J Parasitol. 2008;3(2):34-7.

17. Assmar M, Amirkhani A, Piazak N, Hovanesian A, Kooloobandi A Etessami R. [Toxoplasmosis in Iran. Results of a seroepidemiological study]. Bull Soc Pathol Exot.1997;90(1):19-21. [PubMed: 9264742].

18. Ghorbani M, Edrissian G, Assad N. Serological survey of toxoplasmosis in the northern part of Iran, using indirect fluorescent antibody technique. Transactions Royal Soc Trop Med Hygiene. 1978;72(4):369-71.

19. Sharif M, Ziaei H, Daryani A, Ajami A. Seroepidemiological study of toxoplasmosis in intellectual disability children in rehabilitation centers of northern Iran. Res Dev Disabil. 2007;28(3):219-24. doi: 10.1016/j.ridd.2006.03.001. [PubMed: 16650733].

20. Kelishadi R, Heshmat R, Motlagh ME, Majdzadeh R, Keramatian K, Qorbani M, et al. Methodology and Early Findings of the Third Survey of CASPIAN Study: A National School-based Surveillance of Students' High Risk Behaviors. Int J Prev Med. 2012;3(6):394-401. [PubMed: 22783465].

21. Daryani A, Sarvi S, Aarabi M, Mizani A, Ahmadpour E, Shokri A, et al. Seroprevalence of Toxoplasma gondii in the Iranian general population: A systematic review and meta-analysis. Acta Tropica. 2014;137:18594. doi: 10.1016/j.actatropica.2014.05.015.

22. Prusa AR, Hayde M, Pollak A, Herkner KR, Kasper DC. Evaluation of the liaison automated testing system for diagnosis of congenital toxoplasmosis. Clin Vaccine Immunol. 2012;19(11):1859-63. doi: 10.1128/CVI.00489-12. [PubMed: 23015644]

23. Ogle LT, Sen A, Pahlke E, Jocelyn L, Kastberg D, Roey S, et al. International Comparisons in Fourth-Grade Reading Literacy: Findings from the Progress in International Reading Literacy Study (PIRLS) of 2001. 2003.

24. Jones JL, Kruszon-Moran D, Wilson M, McQuillan G, Navin T, McAuley JB. Toxoplasma gondii infection in the United States: seroprevalence and risk factors. Am J Epidemiol. 2001;154(4):357-65. [PubMed: 11495859].

25. Ayi I, Edu SA, Apea-Kubi KA, Boamah D, Bosompem KM, Edoh D. Sero-epidemiology of toxoplasmosis amongst pregnant women in the greater accra region of ghana. Ghana Med J. 2009;43(3):107-14. [PubMed: 20126322].

26. Aspock H, Pollak A. Prevention of prenatal toxoplasmosis by serological screening of pregnant women in Austria. Scandinavian J Infect Dis Suppl.1992:32.

27. Studenicova C, Ondriska F, Holkova R. [Seroprevalence of Toxoplasma gondii among pregnant women in Slovakia]. Epidemiol Mikrobiol Imunol. 2008;57(1):8-13. [PubMed: 18318393].

28. Jaqueti J, Hernandez-Garcia R, Nicolas D, Martinez-Hernandez D, Navarro-Gallar F, Garcia-Esteban RJ. [Serology against Toxoplasma gondii in pregnant women. Development of prevalence rates in the course of 4 years]. Rev Clin Esp. 1991;188(6):278-80. [PubMed: 1780527].

29. Akyar I. Seroprevalence and Coinfections of Toxoplasma gondii in Childbearing Age Women in Turkey. Iran J Public Health. 2011;40(1):637. [PubMed: 23113056]. 
30. Fuente MC, Bovone NS, Cabral GE. [Prophylaxis of prenatal toxoplasmosis]. Medicina (B Aires).1997;57(2):155-60. [PubMed: 9532825].

31. Fuente MC, Bovone NS, Cabral GE. Profilaxis de la toxoplasmosis prenatal. Medicina (Buenos Aires). 1997;57:155-60.

32. Walton BC, De Arjona I, Benchoff BM. Relationship of Toxoplasma antibodies to altitude. Am J Trop Med Hyg. 1966;15(4):492-5. [PubMed: 5328733].

33. Ades AE, Parker S, Gilbert R, Tookey PA, Berry T, Hjelm M, et al. Maternal prevalence of Toxoplasma antibody based on anonymous neonatal serosurvey: a geographical analysis. Epidemiol Infect. 1993;110(1):12733. [PubMed: 8432316].

34. Saffar MJ, Ajami A. Prevalence of toxoplasma gondi in pregnancy in Sari 1376-1377. J Mazandaran Univ Med Sci.1999;9(24):1-5.

35. Foroutan-Rad M, Khademvatan S, Majidiani H, Aryamand S, Rahim
F, Malehi AS. Seroprevalence of Toxoplasma gondii in the Iranian pregnant women: A systematic review and meta-analysis. Acta Trop. 2016;158:160-9. doi: 10.1016/j.actatropica.2016.03.003. [PubMed: 26952970].

36. Gyang VP, Akinwale OP, Lee YL, Chuang TW, Orok A, Ajibaye O, et al Toxoplasma gondii infection: seroprevalence and associated risk factors among primary schoolchildren in Lagos City, Southern Nigeria. Rev Soc Bras Med Trop. 2015;48(1):56-63. doi: 10.1590/0037-8682-03102014. [PubMed: 25860465].

37. Yentur Doni N, Simsek Z, Gurses G, Yildiz Zeyrek F, Demir C. Prevalence and associated risk factors of Toxoplasma gondii in female farmworkers of southeastern Turkey. J Infect Dev Ctries. 2015;9(1):87-93. doi 10.3855/jidc.5824. [PubMed: 25596576]. 